\title{
FULTON V. CITY OF PHILADELPHIA: OTRO NUEVO CAPÍTULO EN EL EJERCICIO DE LA LIBERTAD RELIGIOSA
}

\author{
María Soledad Riccardi \\ Abogada, Pontificia Universidad Católica Argentina \\ Master of Laws (LL.M.), Georgetown University Law \\ Contacto: msriccardi@gmail.com
}

Recibido: 6 de agosto de 2021

Aprobado: 20 de septiembre de 2021

Para citar este artículo:

Riccardi, M. S. (2021). "Fulton v. City of Philadelphia: Otro nuevo capítulo en el ejercicio de la libertad religiosa".

Prudentia Iuris, N. 92, pp. 251-265

DOI: https://doi.org/10.46553/prudentia.92.2021.pp.251-265

\begin{abstract}
Resumen: El presente artículo versa sobre la sentencia Fulton et al. v. City of Philadelphia, Pennsylvania, et al., de reciente emisión por parte de la Corte Suprema norteamericana, en relación a la cláusula de libertad religiosa de la Constitución de ese país. En los hechos, la ciudad de Filadelfia planteó discontinuar un contrato que mantenía con "Catholic Social Services" - una agencia de adopción encargada de realizar la evaluación de los potenciales padres adoptivos, con la que la ciudad trabajaba- tras conocerse que esta se negaba a derivar a los niños a parejas del mismo sexo. En su examen, la Corte tuvo que determinar si las leyes involucradas eran neutrales y de aplicación general, de acuerdo con el (controvertido) precedente que rige esta clase de litigios.
\end{abstract}

Palabras clave: Libertad religiosa, Objeción de conciencia, Primera Enmienda, Igualdad, Discriminación. 


\title{
Fulton v. City of Philadelphia:
}

\section{Another new chapter in the exercise of religious freedom}

\begin{abstract}
This article reviews the case Fulton et al. v. City of Philadelphia, Pennsylvania, et al., recently issued by the US Supreme Court, on the free exercise of religion clause of the US Constitution. The case arose after the city of Philadelphia posited the discontinuance of a contract held with "Catholic Social Services", an adoption agency commissioned to certify prospective foster parents, with which the city worked, when it learned that the agency would not certify same-sex couples. In its analysis, the Court had to determine whether the laws involved were neutral and generally applicable, according to the (controversial) precedent which governs such kind of First Amendment litigation.
\end{abstract}

Keywords: Religious exercise, Conscientious objection, First Amendment, Equality, Discrimination.

\section{Fulton v. Città di Philadelphia:}

\section{Un altro nuovo capitolo nell'esercizio della libertà religiosa}

Sommario: Questo articolo si occupa della recente sentenza della Corte Suprema degli Stati Uniti Fulton et al. v. City of Philadelphia, Pennsylvania, et al., in relazione alla clausola della libertà religiosa della Costituzione degli Stati Uniti. Nel caso, la città di Filadelfia ha cercato di interrompere un contratto che aveva con Catholic Social Services - un'agenzia di screening delle adozioni con cui la città ha lavorato- dopo che si è saputo che l'agenzia ha rifiutato di indirizzare i bambini a coppie dello stesso sesso. Nel suo esame, la Corte ha dovuto determinare se le leggi coinvolte erano neutre e di applicazione generale, in conformità con i precedenti (controversi) che regolano questo tipo di controversie.

Parole chiave: Libertà religiosa, Obiezione di coscienza, Primo emendamento, Uguaglianza, Discriminazione, Uguaglianza, Discriminazione. 
FULTON V. CITY OF PHILADELPHIA: OTRO NUEVO CAPÍTULO...

\section{Introducción}

La Primera Enmienda de la Constitución norteamericana, centinela de una serie de derechos, comienza con una redacción simple: "El Congreso no hará una ley que establezca una religión oficial, ni que prohíba su libre ejercicio [...]"1. A pesar de su contundencia y aparente simpleza, la interpretación jurídica sobre el alcance de esta cláusula, usualmente llamada "de libre ejercicio (de la religión)", dista de ser pacífica, especialmente en los tiempos que corren.

Ya en el año 1990, el más alto tribunal de ese país emitía la sentencia Employment Division, Department of Human Resources of Oregon v. Smith ${ }^{2}$, que establecía un control de constitucionalidad demasiado laxo frente a leyes que afectasen el ejercicio de la libertad religiosa. Tal fue el descontento social frente a ese pronunciamiento, que el Congreso respondió aprobando la "Religious Freedom Restoration Act" prácticamente por unanimidad. Esa ley -y tantas otras que le siguieron ${ }^{3}$ - buscó establecer un control de escrutinio estricto para esta clase de controversias demostrando, simultáneamente, el compromiso con la libertad religiosa de la sociedad norteamericana ${ }^{4}$.

En la actualidad, "Smith" se mantiene vigente, aunque con inclaudicables críticas, tanto de parte de comentaristas de la jurisprudencia de la Corte, como de algunos de los miembros que componen el tribunal, quienes

1 "Congress shall make no law respecting an establishment of religion, or prohibiting the free exercise thereof [...]”. Traducción libre. Todas las traducciones presentadas en este artículo son propias, de fuentes no oficiales, salvo indicación en contrario.

2494 U.S. 872 (1990). En adelante, "Smith". En los hechos, dos miembros de la Iglesia Nativa Americana habían consumido peyote, una droga alucinógena, en el marco de una ceremonia religiosa. Como resultado de esa conducta, fueron despedidos de sus trabajos. En razón de ello, solicitaron una compensación por desempleo frente al Estado de Oregon, la que fue denegada, pues se entendió que el motivo de su despido se relacionaba con una mala conducta laboral. La Corte debió entonces decidir si un Estado podía denegar beneficios de desempleo a quienes habían utilizado drogas ilegales con fines sacramentales o, en términos legales, si un individuo podía exceptuarse de cumplir con una ley válida por motivos religiosos.

3 Habitualmente denominada por su acrónimo, la "RFRA" buscó imponer un estándar riguroso de control constitucional sobre aquellas acciones del gobierno que gravasen de manera sustancial el ejercicio de la libertad religiosa. Tras el caso City of Boerne v. Flores, 521 U.S. 507 (1997), "RFRA no longer applies to states or localities but continues to constrain federal government action. Many states, however, have passed their own versions of RFRA that apply to state and local laws of general applicability”. Cfr. Congressional Research Service (CRS). (2020). The Religious Freedom Restoration Act: A Primer. Fecha de consulta: 1-8-2021, de Congressional Research Service. Disponible en: https://crsreports.congress.gov/product/pdf/ IF/IF11490.

4 "[...] Congress, with virtual unanimity, expressed the view that Smith's interpretation is contrary to our society's deep-rooted commitment to religious liberty". Fulton et al. v. City of Philadelphia, Pennsylvania, et al. Ver infra, en nota 5, voto de la mayoría, p. 11. 
apuntan a la necesidad de contar con un marco jurídico que brinde mayor protección, en consonancia con la propia prescripción textual de la Primera Enmienda ${ }^{5}$. Los recientes litigios que se han suscitado en el marco de esta libertad abarcan desde acciones entabladas en el marco de la narrativa de las llamadas "guerras culturales", hasta las restricciones derivadas de la pandemia provocada por el COVID-196.

Fulton et al. v. City of Philadelphia, Pennsylvania, et al. ${ }^{7}$, el caso aquí en análisis, se ocupa de determinar qué es neutral y qué es de aplicación general para los propósitos del examen constitucional impuesto por "Smith" en relación a la cláusula mencionada. En él, una agencia de adopción católica que trabajaba en cooperación con la ciudad de Filadelfia, en el estado de Pennsylvania, se vio obligada, merced de una decisión de la ciudad, a decidir entre dejar de prestar sus servicios -desatendiendo así su misión de servir a los niños necesitados-, o bien, hacerlo de una manera contraria a sus convicciones.

Puntualmente, la ciudad de Filadelfia le informó a la agencia -llamada "Catholic Social Services"- que debía certificar como futuros adoptantes a parejas del mismo sexo en el marco del proceso de adopción, sin perjuicio de que ninguna pareja del mismo sexo había solicitado la certificación a "CSS", y que existían otras agencias en la ciudad que estaban dispuestas a realizarla.

Frente a la negativa de CSS y el consecuente litigio constitucional, se presentan a continuación los argumentos vertidos por las partes, la estructura argumental que la Corte utilizó para decidir el caso, y las críticas expuestas en los votos de los jueces Barrett, Alito y Gorsuch, junto con algunos comentarios relacionados con los conflictos encuadrados en la libertad religiosa y la no discriminación y la incidencia de la libertad de expresión en esta clase de casos ${ }^{9}$.

5 Puntualmente, los argumentos en base al texto legal fueron presentados por el juez Alito en su voto concurrente. No obstante ello, Gorsuch y Barrett también han sido críticos, en sus respectivos votos, del contenido y la vigencia de "Smith".

6 En referencia a los casos sobre libertad religiosa que se desarrollaron en el marco de la pandemia, se pueden citar, entre otros: South Bay United Pentecostal Church, et al. v. Gavin Newsom, Governor of CA, et al., 592 U.S. ___ (2021); Tandon v. Newsom, 593 U.S.___ (2021); The Roma Catholic Archbishop of Washington v. Bowser, Case No. 20-cv-03625 (TNM) (D.D.C. Mar. 25, 2021).

7593 U. S. _ (2021). En adelante, "Fulton".

8 En adelante, "CSS".

9 El presente artículo se desarrolló en el marco del proyecto IUS de investigación titulado "Libertad religiosa: la objeción de conciencia institucional o el derecho de autonomía de las instituciones y el acomodamiento razonable. Una mirada desde la jurisprudencia nacional y el derecho comparado" (2019-2021), dirigido por la Dra. Débora Ranieri de Cechini y co-dirigido por la Lic. María Inés Franck. 


\section{Los hechos. El caso “Smith" como preámbulo}

En relación a los hechos, la controversia planteada en "Fulton" ocurrió en el marco del sistema de servicios sociales para niños huérfanos de la ciudad de Filadelfia, el cual se sustenta en la cooperación con agencias privadas: la ciudad firma contratos anuales con estas para que sitúen a los niños con familias adoptivas y certifiquen a las potenciales familias dentro de los parámetros legales provistos por la normativa estatal ${ }^{10}$. CSS prestó estos servicios, de acuerdo con la misión de la Iglesia Católica de servir a los niños necesitados, durante más de cincuenta años ${ }^{11}$. Como parte de sus creencias religiosas, CSS sostiene que el matrimonio es la unión sagrada entre un hombre y una mujer y, en consecuencia, se niega a certificar como posibles familias adoptivas a parejas heterosexuales que no se encuentren casadas, y a parejas del mismo sexo.

Al momento en que se entabló la acción, ninguna pareja del mismo sexo había solicitado la certificación de la CSS. No obstante ello, en el año 2018, tras la publicación en un periódico de una historia que recopilaba la posición de la Archidiócesis de Filadelfia en el sentido expuesto, la ciudad comenzó una investigación que culminó en la decisión de revisar los vínculos contractuales con CSS y de no derivarle más niños para la tramitación de adopción, salvo que esta aceptase certificar a parejas del mismo sexo.

Respecto a lo decidido por la ciudad, se alegó que la negativa de certificación de estas parejas por parte del CSS infringía una cláusula de no discriminación en el contrato firmado con la agencia y, además, los requisitos de no discriminación contenidos en una regulación local, la "Fair Practices Ordinance".

En este contexto, CSS y un grupo de padres accionaron contra la ciudad, argumentando que las decisiones de esta última violaban las cláusulas de libre ejercicio y de libertad de expresión de la Primera Enmienda.

Para una comprensión más acabada del caso y de los debates que se suscitaron a su alrededor, resulta inevitable referirse al caso "Smith"12. En él, la Corte Suprema sostuvo que aquellas leyes que cargan o gravan inci-

10 Previo a certificar a una familia, las agencias deben llevar a cabo un estudio en el cual deben considerar los criterios legales (en este caso, regulatorios), incluyendo "la habilidad de la familia de proveer cuidado, educación, y supervisión a los niños, las relaciones familiares existentes, y la habilidad para trabajar en conjunto con la agencia”. Esta deberá decidir si aprueba, desaprueba, o aprueba provisoriamente a la familia adoptante. 55 Pa. Code, secciones 3700.64 y 3700.69 (2021).

11 La sentencia menciona que la Iglesia Católica ha asistido a los niños necesitados de Filadelfia, de una forma u otra, por más de dos siglos. "Fulton”. Ob. cit., voto de la mayoría, p. 1.

12 Ver, supra, en 3. 
dentalmente a las religiones no deben, en principio, sujetarse a un escrutinio constitucional estricto bajo la cláusula de libre ejercicio, en la medida en que estas sean "neutrales y de aplicabilidad general" ("neutral and generally applicable").

En otras palabras, la Corte redujo el alcance de la libertad religiosa: impuso un estándar de examen constitucional usualmente denominado rational basis ${ }^{13}$, que resulta mucho más permeable que el escrutinio estricto, y reservó este último a los casos en que las leyes no sean neutrales y de aplicabilidad general (es decir, a leyes que apunten contra la libertad religiosa de manera palmaria o expresa).

Efectuada esta aclaración, en primera instancia la corte interviniente entendió que tanto el contrato como la regulación estatal eran normas neutrales y de aplicabilidad general conforme a "Smith". En la siguiente instancia, la Corte de Apelaciones del Tercer Circuito ratificó lo expuesto y, por cuanto el contrato ya había finalizado, examinó si la ciudad podía incluir nuevos términos en relación al lenguaje que prohibía la discriminación por la orientación sexual como condición para la renovación del contrato. CSS y los padres procedieron con la apelación, solicitando, además, a la Corte Suprema que reconsidere el examen impuesto en "Smith".

\section{La opinión de la Corte}

Los postulados legales presentados por la ciudad se centraron en el alcance de las disposiciones contractuales y regulatorias a las cuales CSS debía cumplimiento. De esta manera, la ciudad sostuvo que la certificación a

13 Existen, en el Derecho norteamericano, tres grandes estándares de análisis constitucional: “- El control de la base racional [o relación racional]: la norma será ratificada si se vincula estrechamente con un interés estatal legítimo. - El control intermedio: la norma será ratificada si se vincula estrechamente con un interés estatal importante. - El control estricto: la norma será ratificada solo si se encuentra diseñada ajustadamente a servir un interés estatal imperioso. Estos no son los únicos estándares que existen, pero los otros exámenes de constitucionalidad pueden entenderse mejor en relación a estos tres. En el caso del control de la base racional, quienes cuestionen la racionalidad de la clasificación legislativa tienen la carga de 'descartar cualquier fundamento imaginable que podría servirle de sustento" ("- Rational Basis Review: the law will be upheld if it is closely related to a legitimate state interest. - Intermediate scrutiny: the law will be upheld if it is closely related to an important state interest. - Strict scrutiny: the law will be upheld only if it is narrowly tailored to a compelling state interest. These are not the only standards of review by any means, but most other tests can be better understood by considering their relationship to these three [...] On rational basis review [...] those attacking the rationality of the legislative classification have the burden to 'negative every conceivable basis which might support it”'). Young, E. A. (2012). The Supreme Court and the Constitutional Structure. Nueva York. Foundation Press. 
realizar por CSS únicamente debía reflejar la adecuación con los requisitos legales previstos en la normativa aplicable.

CSS, por su parte, declaró que emitir una certificación equivalía, para ellos, a extender su aprobación o refrenda a la familia certificada. Es por ello que las acciones de la ciudad habían perjudicado ("have burdened") su ejercicio de la libertad religiosa, al obligarla a dejar de actuar como agencia para la ciudad, o bien, a aprobar relaciones que resultan inconsistentes con sus creencias.

Previo a desarrollar los argumentos de la Corte relacionados con las normas legales involucradas, se adelanta que esta sostuvo que "Fulton" no cae dentro del perímetro de "Smith", pues la ciudad de Filadelfia menoscabó la libertad religiosa de CSS a través de normas que no cumplen con los requisitos de ser neutrales y de aplicabilidad general. Específicamente, se recordó que el Estado no actúa de manera neutral si procede de una manera intolerante con las creencias religiosas, o restrictiva de sus prácticas por su naturaleza religiosa ${ }^{14}$, y que una ley no posee aplicabilidad general si prohíbe una conducta religiosa pero permite, al mismo tiempo, una conducta secular que socava los intereses del Estado de una manera similar.

Una vez establecido el marco dentro del cual se examinó el caso, cabe referirse a las disposiciones contractuales traídas a colación por la ciudad. Esta última había argumentado que las prácticas de CSS infringían la Sección 3.21 de su contrato estándar de servicios de adopción ${ }^{15}$. La Corte, como se anticipó, concluyó que esa disposición no es de aplicabilidad general, como requiere "Smith".

Puntualmente, la sección 3.21 especifica, en su parte relevante, que una agencia debe proveer "servicios" a futuros adoptantes sin considerar su orientación sexual. Además, incorpora un sistema de excepciones individuales, las cuales se encuentran disponibles a la "sola discreción" del Comisio-

14 "Government fails to act neutrally when it proceeds in a manner intolerant of religious beliefs or restricts practices because of their religious nature". "Fulton". Ob. cit., voto de la mayoría, p. 5, con cita de: Masterpiece Cakeshop, Ltd. v. Colorado Civil Rights Comm'n, 584 U. S. _ (2018). En este caso, un pastelero llamado Jack. C. Phillips había sido condenado por discriminación en razón de lo previsto en una ley del Estado de Colorado, tras negarse a realizar un pastel para una ceremonia religiosa entre personas del mismo sexo en virtud de que ello violentaba sus creencias religiosas. La Corte Suprema falló a favor de Phillips, quien había invocado la Primera Enmienda pues entendía que su quehacer se encontraba informado por sus creencias religiosas.

15 Cuya redacción disponía: "Rejection of Referral. Provider shall not reject a child or family including, but not limited to, [...] prospective foster or adoptive parents, for Services based upon [...] their [...] sexual orientation [...] unless an exception is granted by the Commissioner or the Commissioner's designee, in his / her sole discretion". "Fulton". Ob. cit., voto de la mayoría, p. 7. 
nado ${ }^{16}$. La ciudad dejó en claro que no tenía ninguna intención de conceder una excepción en este caso, pero la Corte señaló que no resulta posible rechazar el otorgamiento de esa excepción a casos de "dificultades religiosas" ("religious hardship") sin la existencia de razones imperiosas.

Así, el máximo tribunal recalcó que la inclusión de un sistema formal de excepciones enteramente discrecionales en la sección 3.21 le quita aplicabilidad general al requisito contractual de no discriminación. Frente a ello, la ciudad alegó que la viabilidad de excepciones en esa sección contractual resultaba irrelevante, por cuanto el comisionado nunca concedió ninguna. Sin embargo, la Corte respondió que la creación de un mecanismo formal para la concesión de excepciones remueve la aplicabilidad general de una norma -sin importar si se han otorgado excepciones-, pues invita al Estado a decidir cuáles razones para no cumplirla son lo suficientemente válidas como para ameritar su solicitud.

Este punto en particular ha sido señalado como una posible victoria para la libertad religiosa. En ese sentido, se ha dicho que, "si resulta cierto que incluso la posibilidad teórica de una excepción habilita un examen de escrutinio estricto, entonces Smith no establece tantas limitaciones. [...] Si la mera posibilidad de una excepción implica que el Estado no posee un interés imperioso en aplicar su norma a ningún litigante en particular, es difícil pensar que un litigante religioso vuelva a perder un caso"17.

Continuando con el análisis contractual, tampoco importó la existencia de otra disposición -la sección 15.1-, que prohibía de manera independiente la discriminación en la certificación de los futuros padres o tutores por motivos de orientación sexual, sin admitir excepciones. Al respecto, la mayoría señaló que el Derecho estatal en materia contractual es claro al establecer que una parte del contrato no puede interpretarse de manera que anule otra parte del mismo documento. Como resultado de ello, se entendió que el contrato, interpretado íntegramente, no contenía requisitos de no discriminación que resultasen de aplicabilidad general.

Luego de referirse a las disposiciones contractuales reseñadas, la Corte se ocupó de revisar la FPO, la cual CSS también habría infringido. Esta prohíbe "denegar o interferir con las acomodaciones públicas de un individuo o discriminar de otra forma en razón de su raza, etnia, color, género, orientación sexual [...] discapacidad, estado civil, estado familiar, dentro de muchas otras categorías protegidas"18.

16 "Commissioner of the Department of Human Services".

17 Movesian, M. "A Narrow Victory for Religious Liberty". First Things, 23-6-2021. Fecha de consulta: 4-8-2021. Disponible en: https://www.firstthings.com/web-exclusives/2021/06/anarrow-victory-for-religious-liberty.

18 "Deny[ing] or interfer[ing] with the public accommodations opportunities of an indi- 
De acuerdo con ello, la ciudad argumentó que las agencias con las que trabaja en ese ámbito constituyen "acomodaciones públicas"19 y que, por lo tanto, se ven impedidas de discriminar en razón de la orientación sexual de los potenciales padres adoptivos.

La Corte determinó que tal argumento carecía de sustento, por cuanto las agencias de servicios de adopción no actúan como acomodaciones públicas cuando certifican familias. Explicó que, como característica común, las acomodaciones públicas deben proveer un beneficio al público en general para que los individuos puedan utilizarlo si así lo desean.

Las certificaciones que las agencias realizan respecto de las familias, por contraste, no se encuentran accesibles con inmediatez para el público: implican una evaluación selectiva y personalizada que se parece muy poco a "ser huésped en un hotel, comer en un restaurante o tomar un autobús"20. Las agencias de adopción deben llevar a cabo un estudio intensivo de los aplicantes en una serie de niveles que no se equiparan con las actividades recientemente mencionadas. De esta manera, concluyó que el proceso de certificación resulta excepcionalmente selectivo, lo cual necesariamente informa la aplicabilidad de la regulación (FPO) mencionada. Los servicios de las agencias de adopción no constituyen una acomodación pública bajo la FPO y, por lo tanto, ella no le resulta aplicable a CSS.

Finalmente, la Corte se refiere al examen que sí se ajusta al caso. En ese orden de ideas, sostuvo que el requisito contractual de no discriminación le imponía una carga al ejercicio de la libertad religiosa de CSS y, además, que este no resultaba de aplicabilidad general. A eso añadió que una política estatal puede sobrevivir al escrutinio estricto solo si promueve "intereses del más alto orden" y se adecúa limitadamente a esos intereses. Dicho en otros términos, si esos intereses pueden alcanzarse de otra manera que no resulte gravosa del ejercicio de esta libertad, debe optarse por esos otros medios.

La ciudad argumentó que sus políticas anti-discriminación persiguen tres objetivos: maximizar el número de adoptantes, proteger a la ciudad

vidual or otherwise discriminat[ing] based on his or her race, ethnicity, color, sex, sexual orientation, [...] disability, marital status, familial status, or several other protected categories”. Phila. Code \$9-1106(1) (2016).

19 El término "acomodación pública" se refiere, en general, a un lugar que ofrece bienes y servicios al público en general. Existen leyes federales y estatales que prohíben la discriminación respecto de determinados grupos en lugares que se enrolen en esa categoría. A través de la "Civil Rights Act of 1964", el gobierno federal prohíbe la discriminación por motivos religiosos en lugares de acomodación pública. Findlaw. (2018). Public Accommodations and Equal Rights. Fecha de consulta: 1-8-2021. Disponible en: https://www.findlaw.com/civilrights/civilrights-overview/public-accommodations-equal-rights.html.

20 "Fulton". Ob. cit., voto de la mayoría, p. 12. 
de responsabilidad legal, y garantizar un trato igualitario para los futuros adoptantes y para los niños. La Corte se preguntó, entonces, no si la ciudad tenía un interés imperioso en aplicar sus políticas de no discriminación de una manera general, sino si tenía tal interés en negarle una excepción a CSS.

Los intereses reivindicados por la ciudad resultaron, en suma, insuficientes: no pudo demostrar cómo es que una excepción en favor de CSS pondría en riesgo los objetivos mencionados pues, en todo caso, la inclusión de CSS en el programa redundaba en un incremento del número de potenciales adoptantes. CSS, por su parte, solo perseguía una acomodación que le permitiera continuar con su vocación de servicio hacia los niños de Filadelfia en forma consistente con sus creencias religiosas, sin buscar imponer sus creencias a terceros.

En virtud de todo lo expuesto, la decisión adoptada por la ciudad de Filadelfia no logró sobrevivir un examen de escrutinio estricto y resultó violatoria de la Primera Enmienda y su cláusula de libre ejercicio de la religión. De esta forma, se recordó que, en esta última, las creencias religiosas -sin importar el credo al que pertenezcan- no necesitan ser aceptables, consistentes o comprensibles a terceros que no profesan esa religión para ameritar la protección que ella consagra.

\section{Los votos concurrentes}

Los tres votos concurrentes - de los jueces Barrett, Alito y Gorsuch, respectivamente- tuvieron, como eje central, la elusión de la mayoría de revisitar lo decidido en "Smith", aunque cada uno de ellos presentó tonalidades particulares.

La jueza Amy Coney Barrett (a cuyo voto adhirieron el juez Kavanaugh y el juez Breyer ${ }^{21}$ ) manifestó, sobre si resultaba necesario derogar "Smith", que ella personalmente encuentra "que los registros históricos son más silenciosos que alentadores en relación a si la generación de los padres fundadores entendió que la Primera Enmienda requería excepciones religiosas a leyes de aplicación general en, al menos, algunas circunstancias. En mi visión, los argumentos textuales y estructurales en contra de Smith son más imperiosos. En términos de texto y de estructura, es difícil de ver por qué la Cláusula de Libre Ejercicio -solitaria entre las liberta-

21 Este último, en todo salvo el primer párrafo del voto, en donde la jueza realiza apreciaciones personales sobre cuál sería el sustento de "Smith". 
FULTON V. CITY OF PHILADELPHIA: OTRO NUEVO CAPÍTULO...

des de la Primera Enmienda- no ofrece más que protección respecto de la discriminación"22.

No obstante ello, manifestó también sus dudas sobre qué debería reemplazar a "Smith". Si bien se asume que se debería aplicar un estándar de escrutinio estricto cuando una ley neutral y de aplicación general implique una carga al ejercicio de la libertad religiosa, la jueza presentó sus vacilaciones sobre instaurar ese régimen, especialmente cuando "las resoluciones de la Corte sobre leyes de aplicabilidad general y otros derechos contenidos en la Primera Enmienda (como la libertad de prensa y de reunión) presentan tantos matices"23.

Sin perjuicio de sus comentarios sobre aquel precedente, concordó con que el caso planteado en "Fulton" se encuadra en un examen de escrutinio estricto y, por lo tanto, no había razones, en su entendimiento, para decidir allí si "Smith" debería o no ser derogado ni, mucho menos, qué debería reemplazarlo.

Los votos que siguieron fueron mucho más contundentes. El juez Alito, por su parte, se centró en explicar por qué "Smith" fue un error. A través de un voto rico en precisiones históricas y argumentales, señaló que la lectura que se hizo de la cláusula de libre ejercicio en "Smith" es incluso inconsistente con la interpretación (constitucional, y legal en general) que proponía el propio juez Scalia, autor de la opinión mayoritaria.

Precisó, además, cuál es el problema con "Smith" -además de que este se aparta de una lectura constitucional "normal y habitual" del texto de la Primera Enmienda-. Su razonamiento, su consistencia con decisiones previas, la aplicabilidad de la regla que estableció, y el desarrollo de la decisión en el tiempo son cuatro aspectos preponderantes que se observan previo a la derogación de un precedente. Según Alito, en esa matriz, ninguna de estas dimensiones aboga por el mantenimiento de lo establecido en esa decisión.

Alito se animó, además, a proponer qué debería reemplazar a "Smith": el estándar previo, derogado por ese propio caso ${ }^{24}$. En ese sentido, una ley que implique una carga sustancial ("substantial burden") al ejercicio reli-

22 "I find the historical record more silent than supportive on the question whether the founding generation understood the First Amendment to require religious exemptions from generally applicable laws in at least some circumstances. In my view, the textual and structural arguments against Smith are more compelling. As a matter of text and structure, it is difficult to see why the Free Exercise Clause -lone among the First Amendment freedoms-offers nothing more than protection from discrimination". "Fulton". Ob. cit., voto de la jueza Barrett, p. 1.

23 "I am skeptical about swapping Smith's categorical antidiscrimination approach for an equally categorical strict scrutiny regime, particularly when this Court's resolution of conflicts between generally applicable laws and other First Amendment rights -like speech and assembly - has been much more nuanced". "Fulton". Ob. cit., voto de la jueza Barrett, pp. 1-2.

24 Sherbet $v$. Verner, 374 U.S. 398 (1963). 
gioso solo debería sobrevivir si se ajusta limitadamente a cumplir un interés estatal imperioso ("narrowly tailored to serve a compelling government interest").

Otra idea expresada por el juez Alito, casi al final de su voto, se relaciona con la expresión de la postura de CSS y lo que ella representa. En relación a ello, señaló que la problemática encarnada en "Fulton" es justamente la expresión de la idea de que parejas del mismo sexo no deberían ser padres adoptivos, en el entendimiento de que el matrimonio consiste en la unión de un hombre con una mujer.

Si bien el juez entiende que esa idea puede no ser compartida por algunas personas hoy en día, no corresponde suprimir derechos contenidos en la Primera Enmienda por ello. Incluso refirió a la propia jurisprudencia sobre libertad de expresión de la Corte, que ha sostenido, reiterada y contundentemente, que en una sociedad pluralista la expresión de una idea no puede suprimirse solo porque algunos la encuentren ofensiva ${ }^{25}$. Asimismo, en comentario a casos anteriores, autores han señalado que quienes disienten con la expresión de una idea no pueden alegar discriminación si otros individuos se rehúsan a cooperar o difundir sus mensajes ${ }^{26}$.

Finalmente, el voto del juez Gorsuch (acompañado por los jueces Thomas y Alito) tuvo otro tenor. Este comienza siendo crítico con "Smith" -"no respetó los precedentes de esta Corte, fue errado en términos del significado público original de la Constitución, y ha demostrado ser inviable en la práctica"27- pero es aún más crítico con lo decidido por la mayoría en "Fulton", tanto en su razonamiento como en sus posibles resultados. De igual manera, es enfático en la necesidad de derogar "Smith" y ve a "Fulton" como una oportunidad perdida en ese camino.

En cuanto al razonamiento en "Fulton", Gorsuch señaló que este es en extremo enrevesado. En primer lugar, el voto mayoritario intentó demostrar que la norma cuestionada de Filadelfia no es de aplicación general -determinación contraria a la expuesta en primera instancia. Explicó que se ignoró la definición expansiva que el FPO da sobre "acomodaciones públicas",

25 "We have covered this ground repeatedly in free speech cases. In an open, pluralistic, self-governing society, the expression of an idea cannot be suppressed simply because some find it offensive, insulting, or even wounding". "Fulton". Ob. cit., voto del juez Alito, p. 74.

26 "[...] freedom of speech and of religion do not entail the right to compel others to support, participate in, or endorse the exercise of the right. Dissenters [...] have no claim of discrimination if other individuals refuse to cooperate in or carry their messages”. Cfr. McConnel, M. W. (2018). "Dressmakers, Bakers, and the Equality of Rights". Standford Public Law Working Paper. Disponible en: https://papers.ssrn.com/sol3/papers.cfm?abstract_id=3128373.

27 “[...] Smith failed to respect this Court's precedents, was mistaken as a matter of the Constitution's original public meaning, and has proven unworkable in practice". "Fulton". Ob. cit., voto del juez Gorsuch, p. 1. 
y se instó a considerar otra ley -una ley sobre acomodaciones públicas del Commonwealth de Pennsylvania. Gorsuch se pregunta, así, por qué se debería ignorar la norma de la ciudad y sí considerar la ley del Commonwealth, para indicar que, en rigor, el voto mayoritario tampoco ofrece un porqué.

La mayoría enfatizó que la ley de Pennsylvania enumera varios ejemplos de acomodación pública; entre ellos, hoteles, restaurantes y piletas de natación. Argumentó que las agencias de adopción, al contrario de los establecimientos mencionados, requieren un proceso de evaluación personalizada y selectiva. Pero Gorsuch señaló que esa distinción no es de carácter legal: no se encuentra ni en el texto de la ley ni en la jurisprudencia del Estado; menos aún en las presentaciones hechas por las partes. Enfatizó que la Corte simplemente lo declara "una nueva norma del common law de Pennsylvania impuesta por la Corte Federal"28.

Encontró, además, fallas en esa lógica, pues la ley incluye, por ejemplo, a las universidades como "acomodaciones públicas", las cuales sí realizan procesos de evaluación personalizada y selectiva, tanto para elegir a sus estudiantes como a sus profesores. Si estas pueden considerarse como acomodaciones públicas en la ley estatal, no queda claro por qué las agencias de adopción no podrían catalogarse de igual manera.

Sobre las disposiciones contractuales señaló, además, que la sección 3.21 contempla excepciones solo para una determinada parte del proceso de adopción; puntualmente, la de derivaciones. Pero el caso, argumenta, se ocupa de una instancia previa a esta: la de reclutamiento y certificación. Y en esas etapas, indica, la prohibición de la sección 15.1 es absoluta.

Finalmente, Gorsuch criticó el uso del principio según el cual, en ese Estado, se deben interpretar las distintas cláusulas de un contrato sin que una de ellas anule otra, ignorando la usual norma interpretativa que indica que una disposición especial puede convivir cómodamente con otra más general. En virtud de ello, la Corte reescribió la sección 15.1, que pasó a contener su propia reserva de discreción paralela.

Atento a lo expuesto, Gorsuch sugirió que la Corte se encontraba, en sus palabras, "tan ansiosa de no decir nada sobre el futuro de Smith, que está dispuesta a decir prácticamente cualquier cosa sobre el derecho municipal y las presentaciones de las partes" 29 .

En cuanto a los resultados, sostuvo que la judicialización, de este caso en particular y de esta clase de casos en general, recién comienza. En este

28 "The majority just declares it - a new rule of Pennsylvania common law handed down by the United States Supreme Court". "Fulton". Ob. cit., voto del juez Gorsuch, p. 3.

29 "Given all the maneuvering, it's hard not to wonder if the majority is so anxious to say nothing about Smith's fate that it is willing to say pretty much anything about municipal law and the parties' briefs". "Fulton". Ob. cit., voto del juez Gorsuch, p. 7. 
pleito, por ejemplo, la ciudad podría simplemente reescribir el contrato, y CSS volvería al comienzo del embrollo, pues fue clara la determinación por parte de la ciudad de Filadelfia: "[...] si CSS no quiere proveer servicios de adopción a familias del mismo sexo, la ciudad prefiere que, directamente, no provea servicios a nadie" 30 .

CSS tampoco será el único afectado, según Gorsuch, por la indecisión de la Corte. Los efectos -y, principalmente, los costos- los sentirán muchos individuos y grupos a lo largo del país: en dinero, en tiempo y en incertidumbre sobre el alcance de sus libertades religiosas. Incluso los jueces deberán esforzarse para entender y aplicar el estándar de "Smith", como resultado de este nuevo pronunciamiento, incluso treinta años después de su emisión.

\section{Conclusión}

Cualquiera sea la próxima sentencia que la Corte norteamericana emita en relación a los derechos aquí en pugna, queda claro que, pese a aquellos que insisten en forzar a la religión como una actividad eminentemente privada o individual, cierto es que esta, como han señalado prominentes autores en la materia, se manifiesta por múltiples canales que encuentran su cauce en la vida en comunidad ${ }^{31}$.

Si bien "Fulton" ha sido considerado como una victoria para la libertad religiosa, se ha advertido también que esta puede ser tanto decisiva como efímera, dependiendo de cómo se aplique a futuro esta decisión ${ }^{32}$. Aunque su resultado final convive armoniosamente con lo prescripto por la cláusula constitucional, "Fulton" no presenta, como se ha expresado a lo largo de este comentario, un razonamiento jurídico evidente o claro, que pueda allanar el camino para los próximos litigantes que necesitan proteger su libertad religiosa. No obstante ello, no puede soslayarse que, en este pronunciamiento, CSS encontró -a través de la Primera Enmienda- el espacio para afirmar su fe con libertad.

30 "If CSS is unwilling to provide foster-care services to same-sex couples, the City prefers that CSS provide no foster-care services at all”. "Fulton". Ob. cit., voto del juez Gorsuch, p. 8 .

31 Garnett, R. W. (2015). "Religious Accommodations and -and among- Civil Rights: Separation, Toleration, and Accommodation”. 88 S. Cal. L. Rev. 493.

32 Ver supra, en nota 17. Ver también Garnett, R. W. "After Fulton, Religious Foster Care Agencies Still Vulnerable”, First Things, 22-6-2021. Fecha de consulta: 4-8-2021. Disponible en: https://www.firstthings.com/web-exclusives/2021/06/after-fulton-religious-foster-careagencies-still-vulnerable. 
FULTON V. CITY OF PHILADELPHIA: OTRO NUEVO CAPÍTULO...

\section{Bibliografía}

Congressional Research Service (CRS) (2020). The Religious Freedom Restoration Act: A Primer, de Congressional Research Service. Disponible para su consulta en: https://crsreports.congress.gov/product/pdf/IF/IF11490

Findlaw (2018). Public Accommodations and Equal Rights, de FindLaw. Disponible para su consulta en: https://www.findlaw.com/civilrights/civil-rights-overview/ public-accommodations-equal-rights.html

Garnett, R. W. (2015). "Religious Accommodations and -and among- Civil Rights: Separation, Toleration, and Accommodation". 88 S. Cal. L. Rev. 493.

Garnett, R. W. (22-6-2021). "After Fulton, Religious Foster Care Agencies Still Vulnerable", First Things. Disponible para su consulta en: https://www.firstthings. com/web-exclusives/2021/06/after-fulton-religious-foster-care-agencies-still-vulnerable

McConnell, M. W. (2018). "Dressmakers, Bakers, and the Equality of Rights". Stanford Public Law Working Paper. Disponible para su consulta en: https://papers. ssrn.com/sol3/papers.cfm?abstract_id=3128373

Movesian, M. (23-6-2021). "A Narrow Victory for Religious Liberty", First Things. Disponible para su consulta en: https://www.firstthings.com/webexclusives/2021/06/a-narrow-victory-for-religious-liberty

Young, E. A. (2012). The Supreme Court and the Constitutional Structure (1 $\left.{ }^{\mathrm{er}} \mathrm{ed}.\right)$. Nueva York. Foundation Press. 